\title{
Diffusion of topological charge and scaling of autocorrelation times in hybrid Monte Carlo simulations of lattice QCD
}

\author{
Greg McGlynn* \\ Columbia University \\ E-mail: gem2128@columbia.edu
}

\section{Robert D. Mawhinney}

Columbia University

\begin{abstract}
We determine the scaling behavior of the autocorrelation times of observables constructed from the topological charge density on lattices with periodic and open boundary conditions using a series of high-statistics numerical simulations. The autocorrelation functions of such observables turn out to obey a simple differential equation which allows the motion of topological charge in hybrid Monte Carlo simulations to be understood in terms of only two processes: diffusion and tunneling. There is a characteristic lattice spacing at which open boundary conditions become worthwhile for reducing autocorrelations and we show how this lattice spacing is related to the diffusion constant, the tunneling rate, and the lattice Euclidean time extent. The subject of this talk was treated in more detail in a paper recently published by the authors [1].
\end{abstract}

The 32nd International Symposium on Lattice Field Theory

23-28 June, 2014

Columbia University New York, NY

\footnotetext{
*Speaker.
} 


\section{Introduction}

As lattice QCD simulations approach the continuum limit, the autocorrelation times of many observables increase. For statistical errors to be fully under control, the total length of a simulation must be much longer than any autocorrelation time, so this represents a danger for modern simulations of QCD which are pushing to finer lattice spacings. The topological charge in particular shows a very rapidly increasing autocorrelation time as the lattice spacing $a$ goes to zero $[2,3,4,5]$. This results from the fact that in a periodic volume the topological charge of a continuum gauge field cannot change along any continuous path through field space. The topological charge of a lattice gauge field can change, but only by going through non-continuum-like configurations, and these configurations become very rare close to the continuum limit. Therefore the simulation becomes trapped in a single topological sector of field space, tunneling between sectors only very rarely.

In order to get around this it has been proposed to switch from periodic boundary conditions to "open" boundary conditions on the gauge field for the Euclidean time direction [6]. In [6] evidence was given that when open boundary conditions are used the integrated autocorrelation time of the topological charge increases like $1 / a^{2}$ as $a \rightarrow 0$, which is a much slower increase than expected from periodic boundary conditions. This suggested that open boundary conditions can produce a dramatic speedup of the topological charge in QCD simulations.

The purpose of the work presented in this talk is to directly compare the scaling behavior of autocorrelations between periodic and open boundary conditions. In [6] only open boundary conditions were simulated, so there was no direct comparison to periodic boundary conditions. In addition to performing this raw numerical comparison we are able to develop a mathematical model of autocorrelation times of topological observables which provides insight into how topological charge moves around the lattice and explains when open boundary conditions are useful for reducing autocorrelations.

\section{Simulations}

We simulate pure SU(3) gauge theory using the DBW2 gauge action at five lattice spacings in the range $0.2 \mathrm{fm} \leq a \leq 0.1 \mathrm{fm}$, keeping the physical volume fixed at $(1.6 \mathrm{fm})^{3} \times 3.2 \mathrm{fm}$. At each lattice spacing we run a pair of simulations, one with periodic and one with open boundaries in the time direction. We use the DBW2 action because it demonstrates the freezing of topology at comparatively coarse lattice spacings, making the phenomenon easier to study [7]. On the periodic lattices, as $a$ is decreased from $0.2 \mathrm{fm}$ to $0.1 \mathrm{fm}$ the autocorrelation time of the topological charge increases by roughly a factor of 100, as shown in Figure 1. This is the expected dramatic slowdown of the topological charge with periodic boundary conditions.

To make a comparison between periodic and open boundaries we need to choose appropriate observables. In particular we need to avoid the region of the lattice immediately adjacent to the open boundaries. The reason is that the presence of the open boundaries distorts the simulated physics near the boundary. We are only interested in whether open boundary conditions can speed up simulations of infinite-volume physics, so we should avoid regions where the physics is not similar to infinite-volume QCD. The effect of the open boundaries on various observables can be 


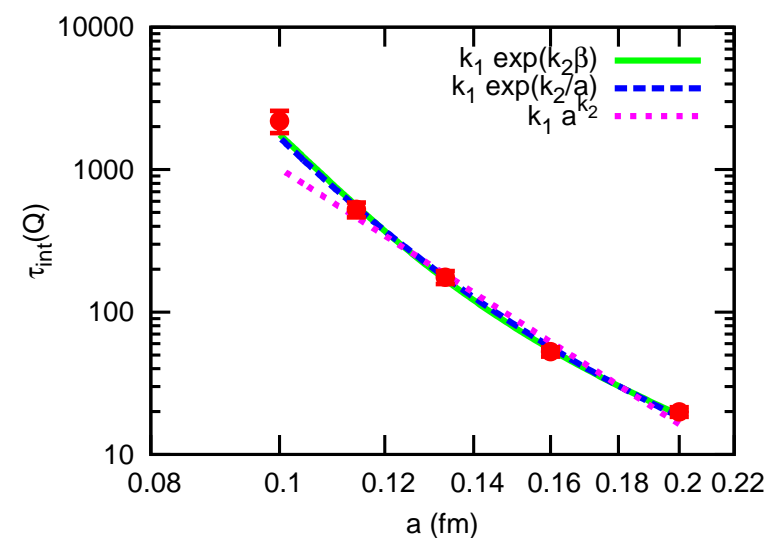

Figure 1: The integrated autocorrelation time of the global topological charge in MD time units on our periodic lattices, with various fits to the data. In the first fit, $k_{2} \approx 20$; in the second fit $k_{2} \approx 0.90 \mathrm{fm}$; in the third fit, $k_{2} \approx-6$.

measured and we find in our simulations that if we stay at least $0.8 \mathrm{fm}$ from the boundary then the boundary effects are negligible.

The basic observable we study is the time slice topological charge $Q(t)$, which is the fieldtheoretic topological charge density summed over the time slice at Euclidean time $t$. From this we can build the subvolume charge $Q\left(t_{1}, t_{2}\right)$, which is the topological charge density summed over the Euclidean time interval $t_{1} \leq t<t_{2}$. On periodic lattices we have the global charge $Q \equiv Q(0, T)$ (but on open lattices the global charge is contaminated by boundary effects).

We collect very high statistics which allows us to precisely measure the integrated autocorrelation times of these observables even when these autocorrelation times are thousands of molecular dynamics time units (MDU). In Figure 2 we plot the measured integrated autocorrelation times of $Q(T / 2)$, the charge on the central time slice, and $Q(T / 4,3 T / 4)$, the charge in the central halfvolume.

We see that, like $Q$ in a periodic volume, these autocorrelation times are rapidly increasing as $a \rightarrow 0$. Furthermore we see that for $a$ greater than some characteristic lattice spacing, here about $0.11 \mathrm{fm}$, open and periodic boundary conditions produce identical autocorrelation times, while for $a$ smaller open boundary conditions start to produce some speedup.

\section{Diffusion model}

Our main result is a mathematical model for topological autocorrelations which explains this scaling behavior. Let $Q(t, \tau)$ denote the topological charge on time slice $t$ at molecular dynamics (MD) time $\tau$. We define the correlation function

$$
C\left(t, t_{0}, \tau\right) \equiv\left\langle Q\left(t, \tau_{0}+\tau\right) Q\left(t_{0}, \tau_{0}\right)\right\rangle
$$

Roughly speaking, this correlation function measures how much topological charge will move from time slice $t_{0}$ to time slice $t$ in an MD time interval $\tau$. We can measure this correlation function to high precision and we find empirically that it obeys a simple differential equation to high accuracy: 


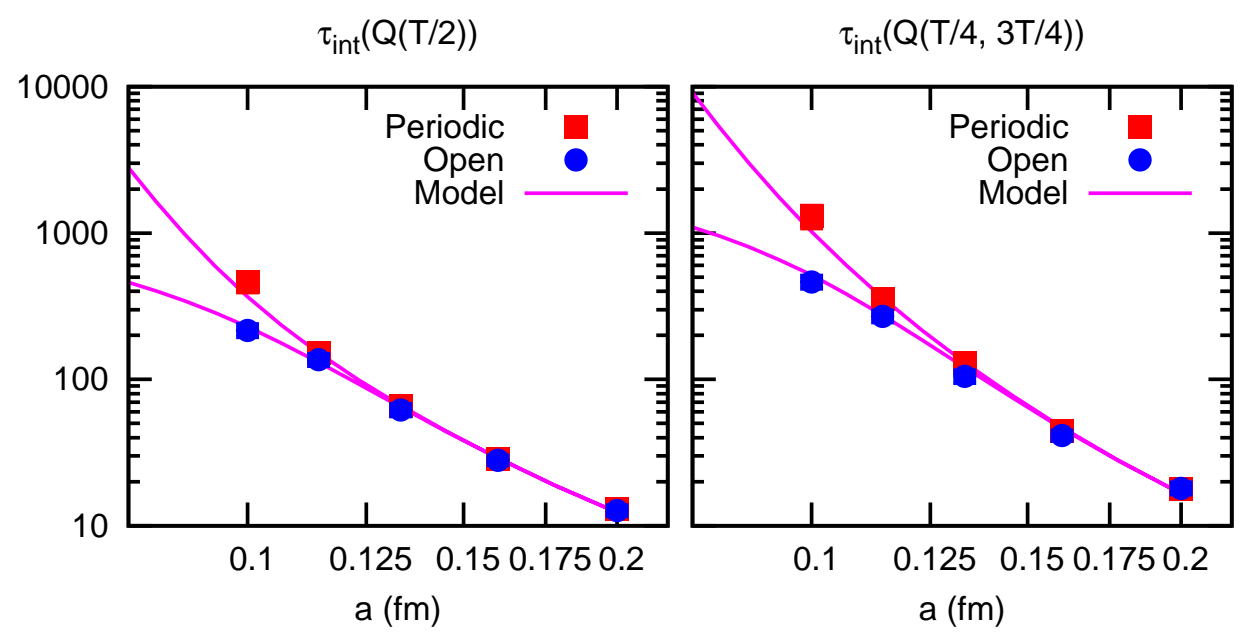

Figure 2: Measurements of the integrated autocorrelation times of the charge on the central time slice and the charge in the central-half volume in MD time units. The "Model" curves are computed using the diffusion model discussed below.

$$
\frac{\partial}{\partial \tau} C\left(t, t_{0}, \tau\right)=\frac{\partial}{\partial t}\left(D(t) \frac{\partial}{\partial t} C\left(t, t_{0}, \tau\right)\right)-\frac{1}{\tau_{\text {tunn }}} C\left(t, t_{0}, \tau\right)
$$

This is a diffusion-decay equation: $D(t)$ is a $t$-dependent diffusion constant. The parameter $\tau_{\text {tunn }}$, which we call the "tunneling timescale," is related to the rate of tunneling between topological sectors. On a periodic lattice it is the same as the integrated autocorrelation time of $Q$. The agreement between Eq. (3.2) and our data is remarkably precise.

Eq. (3.2) applies to both periodic and open lattices. The boundary conditions on the gauge field determine the boundary conditions on $C$ : on periodic lattices $C\left(t, t_{0}, \tau\right)$ is periodic in both $t$ and $t_{0}$. On open lattices $C\left(t, t_{0}, \tau\right)$ goes to zero when either $t$ or $t_{0}$ is at a lattice boundary.

We use a fitting procedure to extract $D(t)$ and $\tau_{\text {tunn }}$ on each of our ensembles. The values obtained are consistent between our periodic and open ensembles except that we find that the diffusion constant $D(t)$ is enhanced in the immediate vicinity of an open boundary. In the bulk $D(t)$ is a constant $D$. As Figure 3 shows, we find that $D$ scales essentially like $a^{2}$. Meanwhile the tunneling timescale $\tau_{\text {tunn }}$ increases roughly like $1 / a^{6}$ as $a \rightarrow 0$, as shown in Figure 1 .

In addition to the tunneling timescale there is also a diffusion timescale. Given a lattice Euclidean time extent $T$ and a diffusion constant $D$, the diffusion timescale is $\tau_{\text {diff }} \equiv T^{2} / 8 D$, which is the MD time to diffuse across half the lattice. This can be regarded as the time it takes topological charge to diffuse into the center of the lattice from the open boundaries. Given the scaling of $D$, $\tau_{\text {diff }}$ scales like $1 / a^{2}$, so that it increases much more slowly than $\tau_{\text {tunn. }}$. As shown in Figure 4 , on coarse lattices $\tau_{\text {tunn }} \ll \tau_{\text {diff }}$ and so we call these lattices "tunneling-dominated." On fine lattices $\tau_{\text {diff }} \ll \tau_{\text {tunn }}$ and we call these lattices "diffusion-dominated."

Figure 4 shows that the transition between the tunneling- and diffusion-dominated regimes is at $a \approx 0.11 \mathrm{fm}$ on our lattices, explaining why open and periodic boundaries start to differ at this $a$ in Figure 2. In the tunneling-dominated regime we do not expect the boundary conditions

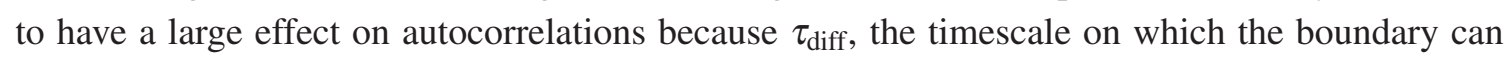




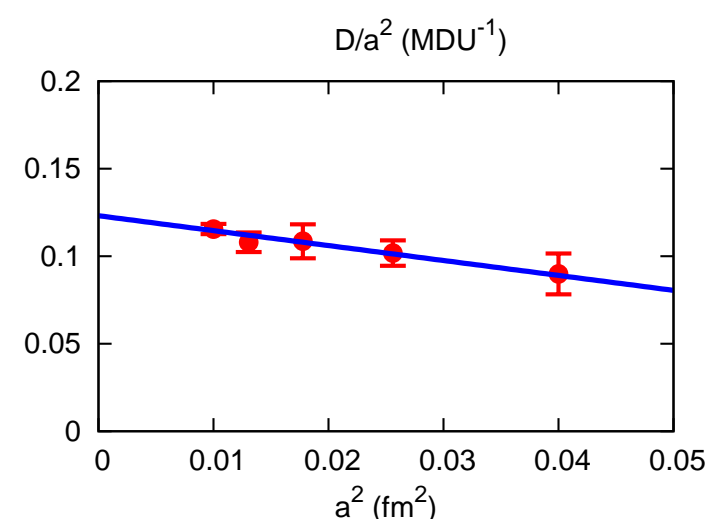

Figure 3: The scaling of the diffusion constant $D$ with $a . D / a^{2}$ is roughly constant. The line is a linear fit to the data.

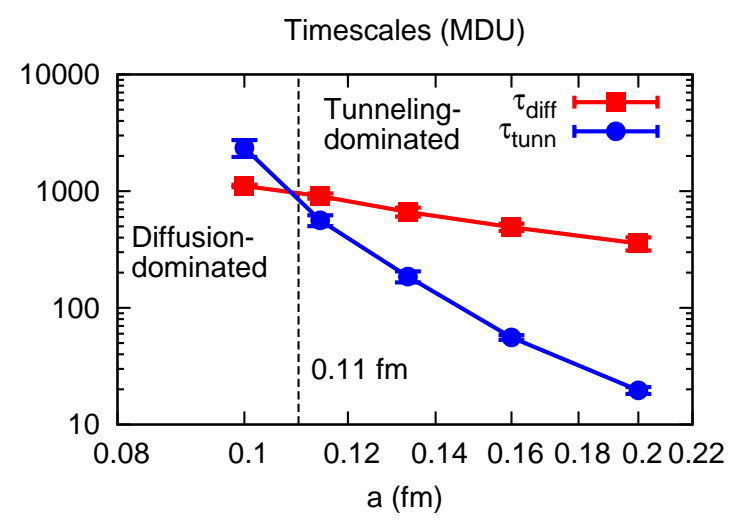

Figure 4: The diffusion and tunneling timescales as a function of $a$.

effect the bulk, is much shorter than $\tau_{\text {tunn }}$, the timescale on which autocorrelations are destroyed by bulk tunneling. But in the diffusion-dominated regime open lattices can decorrelate much faster than periodic lattices by creating and destroying topological charge at the boundaries and allowing these changes to diffuse into the bulk.

The tunneling timescale $\tau_{\text {tunn }}$ depends on the details of the lattice action; it is well-known that some lattice actions are more favorable than others for topological tunneling. The diffusion timescale is probably less sensitive to the lattice action but does depend on the lattice Euclidean time extent $T$. Thus the lattice action and the time extent influence the location of the transition between the tunneling-dominated and diffusion-dominated regimes.

This qualitative picture can be further sharpened by using Eq. (3.2) to actually calculate integrated autocorrelation times. For example we obtain the model curves in Figure 2 by numerically Eq. (3.2) using the known $a$-dependence of $D$ and $\tau_{\text {tunn }}$ as inputs. The model curves fit the data quite well. In some limits we can use Eq. (3.2) to calculate integrated autocorrelation times analytically. For example, we find for the integrated autocorrelation time of $Q(T / 2)$ in various limits: 


$$
\begin{aligned}
\tau_{\text {int }} & =\sqrt{\frac{\pi \sigma^{2} \tau_{\text {tunn }}}{2 D}} & & \text { (tunneling-dominated limit) } \\
\tau_{\text {int }} & =\sqrt{\frac{\pi}{8} \frac{\sigma T}{D}} & & \text { (diffusion-dominated limit, open boundaries) } \\
\tau_{\text {int }} & =\sqrt{2 \pi} \frac{\sigma}{T} \tau_{\text {tunn }} & & \text { (diffusion-dominated limit, periodic boundaries) }
\end{aligned}
$$

Here $\sigma$ is a length scale of about $0.2 \mathrm{fm}$ (see [1]). This tells us that in the diffusion-dominated limit this integrated autocorrelation time scales like $1 / D \sim 1 / a^{2}$ for open boundaries, but like $\tau_{\text {tunn }} \sim 1 / a^{6}$ for periodic boundaries. It turns out that in this limit these scaling laws are shared by all observables built from $Q(t)$, for example the half-volume charge $Q(T / 4,3 T / 4)$.

\section{Conclusions}

We have shown that the autocorrelations of topological observables in lattice QCD simulation are well-described by a simple mathematical model, Eq. (3.2), which incorporates only topological tunneling and diffusion of topological charge. Using the diffusion model we find that at fine enough lattice spacings, the autocorrelation times of topological observables grow like $1 / a^{2}$ on open lattices but grow much faster, perhaps like $1 / a^{6}$, on periodic lattices. The diffusion model identifies two characteristic timescales of QCD simulations, the tunneling timescale $\tau_{\text {tunn }}$ and the

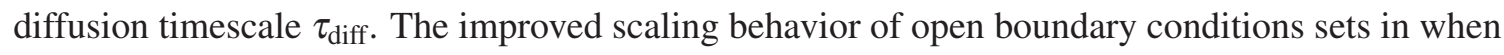
the lattice spacing is fine enough that $\tau_{\text {diff }} \ll \tau_{\text {tunn }}$. The lattice spacing at which this occurs depends on the lattice action and the Euclidean time extent. For further details about the work reported here we refer the reader to [1].

\section{References}

[1] G. McGlynn and R. D. Mawhinney, "Diffusion of topological charge in lattice QCD simulations," Phys. Rev. D 90, 074502 (2014) [arXiv:1406.4551 [hep-lat]].

[2] S. Schaefer et al. [ALPHA Collaboration], "Critical slowing down and error analysis in lattice QCD simulations," Nucl. Phys. B 845, 93 (2011) [arXiv:1009.5228 [hep-lat]].

[3] L. Del Debbio, H. Panagopoulos and E. Vicari, “ $\theta$ dependence of SU(N) gauge theories,” JHEP 0208, 044 (2002) [hep-th/0204125].

[4] M. Lüscher, "Topology, the Wilson flow and the HMC algorithm," PoS LATTICE 2010, 015 (2010) [arXiv:1009.5877 [hep-lat]].

[5] M. Lüscher, "Step scaling and the Yang-Mills gradient flow," JHEP 1406, 105 (2014) [arXiv:1404.5930 [hep-lat]].

[6] M. Lüscher and S. Schaefer, "Lattice QCD without topology barriers," JHEP 1107, 036 (2011) [arXiv:1105.4749 [hep-lat]].

[7] Y. Aoki, T. Blum, N. Christ, C. Cristian, C. Dawson, T. Izubuchi, G. Liu and R. Mawhinney et al., "Domain wall fermions with improved gauge actions," Phys. Rev. D 69, 074504 (2004) [hep-lat/0211023]. 